\title{
Low-Temperature Structure of Indium Quantum Chains on Silicon
}

\author{
C. Kumpf, ${ }^{1}$ O. Bunk, ${ }^{2}$ J. H. Zeysing, ${ }^{2}$ Y. Su, ${ }^{2}$ M. Nielsen, ${ }^{1}$ R. L. Johnson, ${ }^{2}$ R. Feidenhans' $1,{ }^{1}$ and K. Bechgaard ${ }^{1}$ \\ ${ }^{1}$ Condensed Matter Physics and Chemistry Department, Risø National Laboratory, DK-4000 Roskilde, Denmark \\ ${ }^{2}$ II. Institut für Experimentalphysik, Universität Hamburg, D-22761 Hamburg, Germany
}

(Received 18 April 2000; revised manuscript received 10 August 2000)

\begin{abstract}
The array of quasi-one-dimensional indium chains in the $\mathrm{Si}(111)-(4 \times 1)$-In surface reconstruction exhibits a phase transition to a low-temperature $(8 \times 2)$ phase. It has been suggested that this phase transition is related to a charge density wave (CDW) formation. The $\mathrm{x}$-ray diffraction results presented here demonstrate that at $20 \mathrm{~K}$ the CDW has not yet condensed into a superstructure even though good transverse coupling was established. This indicates that CDW formation cannot be the driving force for the phase transition. Furthermore we elucidate the subtle highly anisotropic interchain correlations and reveal the detailed atomic structure of the low-temperature $(8 \times 2)$ phase.
\end{abstract}

PACS numbers: 68.35.Bs, 68.35.Rh, 68.65. $+\mathrm{g}$

Low-dimensional systems exhibit a variety of exotic physical phenomena such as charge density waves (CDWs), Peierls instabilities, or the formation of non-Fermi-liquid-like ground states [1-6]. Self-organized adsorbate-induced modification of semiconductor surfaces is a powerful technique for fabricating such lowdimensional nanoscale quantum structures. Here we report on the formation of a periodic lattice distortion found on the $\operatorname{Si}(111)-(4 \times 1)$-In surface as the system undergoes a temperature-induced phase transition to a $(8 \times 2)$ reconstruction.

Adsorption of indium on $\mathrm{Si}(111)$ produces a variety of surface reconstructions [7] that are semiconducting for coverages below one monolayer and metallic at larger coverages. As first reported by Lander and Morrison in 1965 [8], a $(4 \times 1)$ phase occurs at the borderline between the two regions. Although this phase has been investigated with a variety of techniques over the past 30 years the definitive structural model was established only recently, using surface $\mathrm{x}$-ray diffraction [9]. The surface structure consists of quasi-one-dimensional indium chains, separated from each other by chains of silicon atoms. Electronically the room temperature (RT) phase exhibits the Fermi-liquid-like behavior of a strongly anisotropic two-dimensional metal $[10,11]$. It was reported that the system undergoes a reversible phase transition accompanied by a $1 \mathrm{D}$ CDW along the indium chains at approximately $100 \mathrm{~K}$ [11]. Reflection high-energy electron diffraction experiments revealed that-due to the phase transition - the periodicity of the surface reconstruction doubles along the chains with little correlation between the chains. Since half-order streaks rather than peaks were observed in the direction along the chains, the new phase was called a ( $4 \times$ "2") reconstruction and it was speculated that the true low temperature (LT) ground state might be a well-ordered $(8 \times 2)$ phase with the CDWs locked in phase [11]. Recent core-level photoemission measurements [12] revealed that the LT phase has metallic character as evidenced by the large asymmetry of the In $4 d$ core-level spectra, but in angle- resolved photoelectron spectroscopy the valence band intensity fell with a power-law dependence and vanished at the Fermi level. It was suggested that this system undergoes a Fermi-liquid to Luttinger-liquid transition rather than a Peierls-like instability [12].

To establish the nature of the phase transition and determine the geometrical structure of the ground state we have investigated the LT phase using surface $\mathrm{x}$-ray diffraction. The measurements performed at $20 \mathrm{~K}$ reveal that the low-temperature phase is an $(8 \times 2)$ reconstruction; the structural change associated with the phase transition is primarily the formation of trimers within the In chains. The structure shows very strong chain to chain correlations only in the direction perpendicular to the chains, i.e., a well-developed $(8 \times)$ superstructure due to transverse correlations. In the direction parallel to the chains diffuse streaks still persist at $20 \mathrm{~K}$, indicating that the longitudinal interchain correlation between neighboring chains is poor - as opposed to the good intrachain correlation within a single chain observed in scanning tunnel microscopy $(\mathrm{STM})$ images [11]. Even at $20 \mathrm{~K}$ the $(8 \times 2)$ superstructure is not fully developed. This key result implies that the phase transition is not driven by a CDW. For a mere Peierls instability one would expect that the CDW should condense into a fully developed superstructure for such a good transverse chain to chain coupling.

The sample was prepared using standard procedures in an ultrahigh vacuum (UHV) system equipped with extensive sample characterization facilities. It was transferred to a UHV cryostat, and after cooling to $20 \mathrm{~K}$ an extended data set was measured at the BW2 beam line at the Hamburg synchrotron radiation laboratory (HASYLAB). The data set consisted of 230 nonequivalent in-plane reflections, two crystal truncation rods, and eight fractional-order rods. Further information on sample preparation, data analysis, and the structural parameters are given in Ref. [13].

The half-order reflections in the $k$ direction were never sharp, even at low temperatures, but only streaks as in Ref. [11]. Strong eighth-order peaks were observed along the $h$ direction so the measured data set had an $(8 \times 1)$ 
symmetry. The intensity distribution of the quarter-order reflections resembles that of the $(4 \times 1)$ RT phase [9], which suggests that the two structures must be similar. A comparison of the contour maps of the 2D-Patterson function (autocorrelation function of the in-plane data) of both structures confirms this assumption and so the $(4 \times 1)$ configuration was used as the starting model for the refinement of the $(8 \times 2)$ structure. The displacements of the atoms are restricted to a glide line in the [11 $\overline{2}]$ direction of the Si substrate, as can be inferred from the missing eighth-order reflections along the $k=0$ line in the LT phase. Refining the positions of the top layer In and $\mathrm{Si}$ atoms and two double layers of the silicon substrate results in $\chi^{2}=3.46$ and indicates that the model contains all of the essential features [14]. The best fit depicted in Fig. 1 (with $\chi^{2}=2.87$ ) was achieved by allowing the DebyeWaller factors of the indium atoms to be adjusted. The corresponding structural model is shown in Fig. 2. The final atomic positions of the LT reconstruction (red and blue circles) are shown, as well as the positions in the RT model (open circles). It can be seen that the phase transition consists mainly of a pairing effect of the In atoms labeled 1,2 and 7,8. This causes a formation of indium trimers (atoms 1-3 and 6-8) and a doubling of the unit cell in the $\mathbf{b}$ direction along the chains. The atomic distances within these trimers are between 2.8 and $3.1 \AA$, close to the indium covalent bond length of $2.9 \AA$. The other two indium atoms 4 and 5 are located very close to T1 on-top positions above the uppermost silicon bulk atoms. The silicon atoms in the top layer (light blue circles) show no discernible in-plane displacements from their RT positions, but a perceptible out-of-plane buckling as can be seen in Figs. $2 b$ and $2 c$. Silicon atoms in deeper layers (dark blue circles) have only small displacements from the RT positions ( $\leq 0.3 \AA$ ), except the two atoms in the second layer marked by small arrows in Figs. $2 \mathrm{~b}$ and $2 \mathrm{c}$ (with displacements of $\approx 0.4 \AA$ ).

The $(8 \times 2)$ unit cell can be subdivided in two $(4 \times 2)$ subcells containing the indium atoms $1-8$ and $1^{\prime}-8^{\prime}$, respectively, as shown in Fig. 2a. The doubling of the $(4 \times 1)$ RT unit cell in the a direction (perpendicular to the chains) is caused by the appearance of a glide line which maps one subcell (atoms 1-8) on the other (atoms $1^{\prime}-8^{\prime}$ ). Sharp eighth-order peaks indicate a strong interchain coupling of the displacements between the neighboring chains in this direction. A correlation length of $\approx 240 \AA$ can be derived for this transverse interchain coupling from the peak width of the eighth-order reflections. The transverse ordering within the $(4 \times 2)$ subcells, calculated from the width of the quarter-order reflections, shows a higher correlation length of $\approx 410 \AA$. Figure 3 shows in-plane line scans through the strongest eighthorder reflections at different temperatures while warming the sample from $20 \mathrm{~K}$ to RT. It can clearly be seen that the peak width of the eighth-order peaks remains constant and the intensity diminishes gradually during the phase
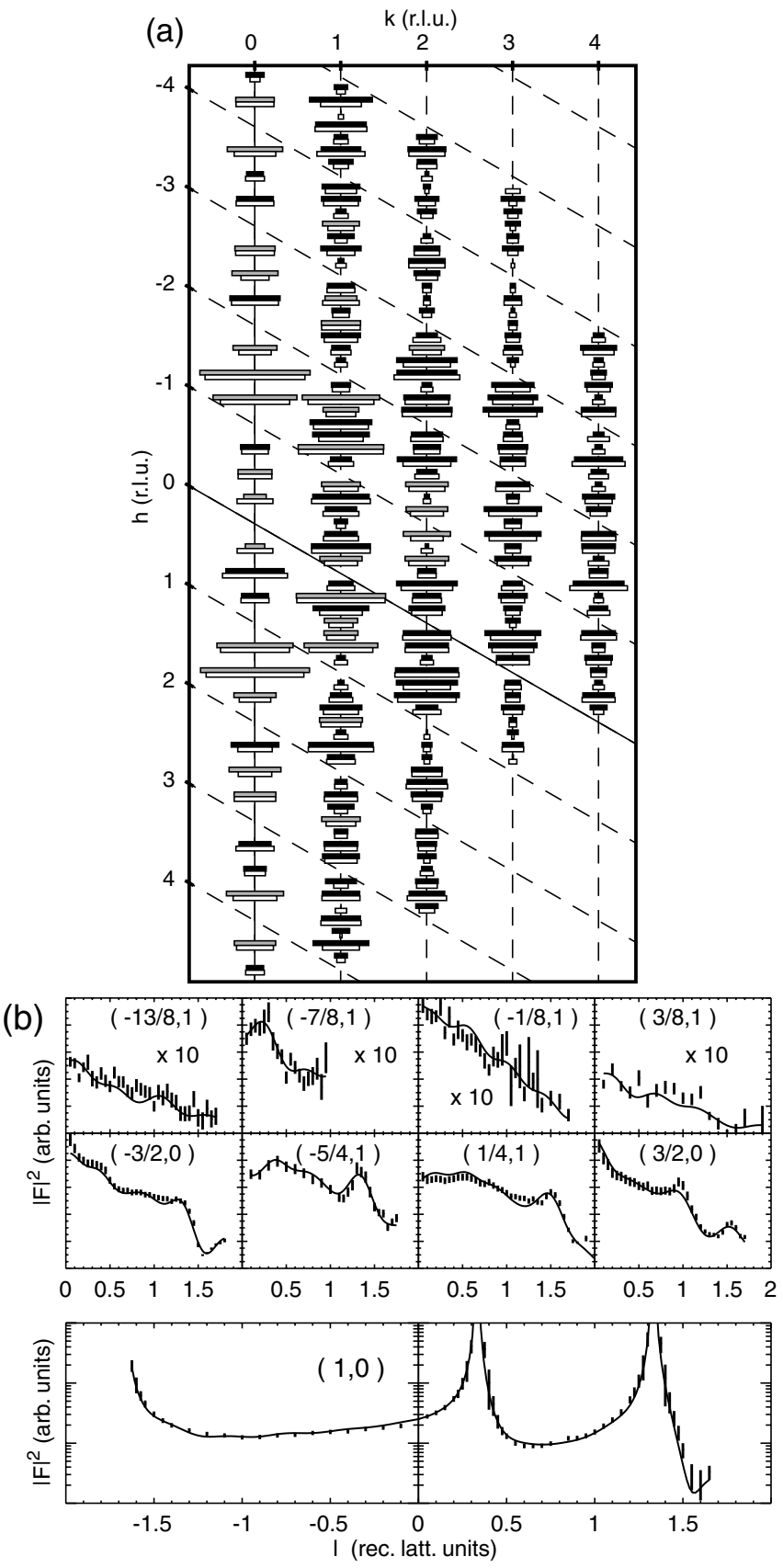

FIG. 1. (a) In-plane data set. The length of the filled and open boxes represent the measured and calculated structure factors. Gray/white boxes have been scaled with a factor of 0.5 . (b) Fractional- and integer-order rod scans.

transition while the intensity of the quarter-order reflection increases. The phase transition is not characterized by a change in correlation length of the interchain coupling or a change in size of the $(8 \times 2)$ domains, but by a steady reduction of the atomic displacements, i.e., a gradual relaxation from the LT to the RT positions.

From a structural point of view the coupling mechanism along the indium chains appears to be unusual. The streaky half-order reflections in the $k$ direction seem to 


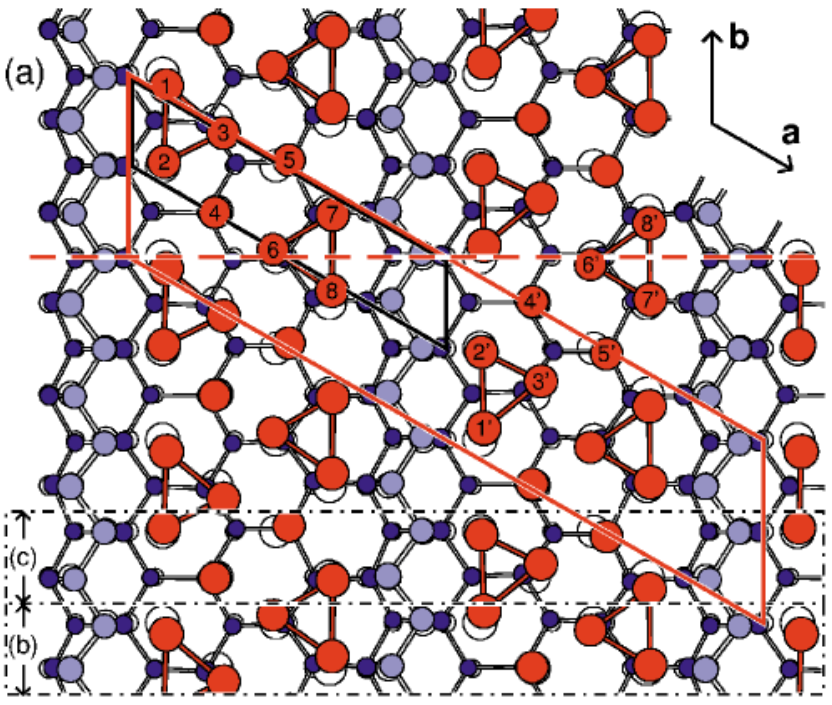

(b)

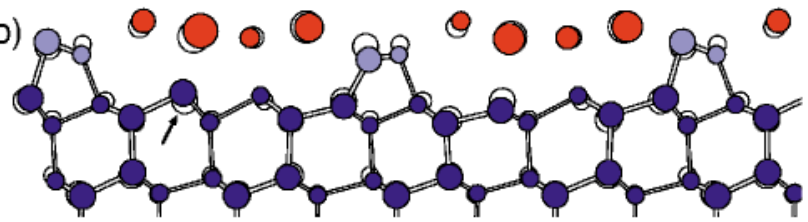

(c)

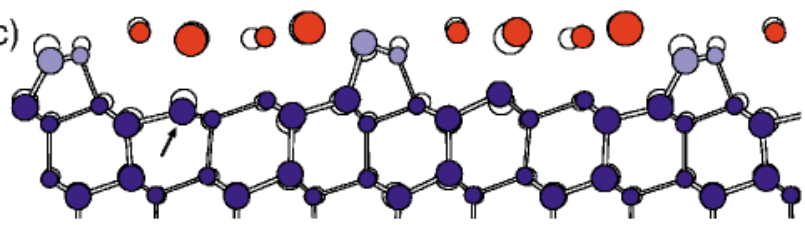

FIG. 2 (color). Model of the Si(111)- $(8 \times 2)$-In LT reconstruction. (a) top and (b),(c) side views. Indium, silicon bulk, and silicon top layer atoms are shown as red, dark blue, and light blue circles, respectively. Open circles mark the atomic positions of the RT $(4 \times 1)$ structure. The $(4 \times 1)$ and $(8 \times 2)$ unit cells are indicated by black and red solid lines. The dashed line indicates the glide line. The regions corresponding to the side views (b),(c) are indicated by dash-dotted lines.

indicate poor long-range order between the indium chains, but the glide-line symmetry makes the situation more complicated. In Fig. 4a the "irreducible unit" of the structure is indicated as a red $(4 \times 2)$ subcell. The magenta unit cell indicates the equivalent part of the structure due

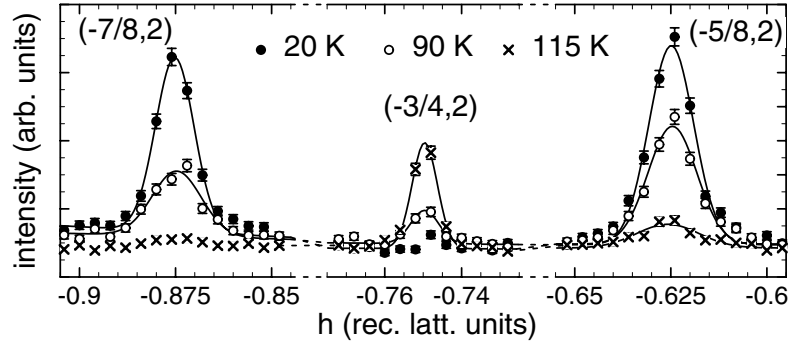

FIG. 3. Line scans through eighth- and quarter-order reflections at different temperatures: at $20 \mathrm{~K}$ (LT phase), at $90 \mathrm{~K}$, and at $115 \mathrm{~K}$ (close to and above the expected transition temperature). The solid lines represent Gaussian fit curves. to glide symmetry (dashed magenta line). With respect to the magenta colored atoms the green atoms in Fig. $4 \mathrm{~b}$ are moved by $[0,1]_{\text {LEED }}$. This atomic configuration can also be described as a $(8 \times 2)$ reconstruction with the same basic structural unit (indicated in red), but a glide line (green dashed line) which is shifted by $\left[0, \frac{1}{2}\right]_{\text {LEED }}$. This modified atomic configuration yields exactly the same $(8 \times 1)$ diffraction pattern and the reconstructions can be distinguished only from the intensities of the half-order reflections in the $k$ direction. This means that the streaky half-order reflections result from poor parallel correlation between pairs of indium chains in the $\mathbf{b}$ direction. In other words, the $(8 \times 2)$ reconstruction exhibits a high degree of " $(4 \times 2)$-subcell disorder," i.e., uncorrelated arrangements of both subcells along the chains. This is in agreement with STM measurements $[11,12]$ and explains the apparent contradiction between the large correlation length along the chains found in STM and streaky diffraction patterns.

The structural features seen in the adlayer at LT have been suggested to be the result of a CDW condensation related to a Peierls instability of the quasi-1D indium chains [11]. Now we proceed to analyze such a model. A priori it is expected that $1 \mathrm{D}$ fluctuations will give rise to diffuse lines related to twice the Fermi wave vector $2 k_{f}$ at temperatures well above the transition, indicating a change of the translation period (e.g., a doubling of the unit cell)
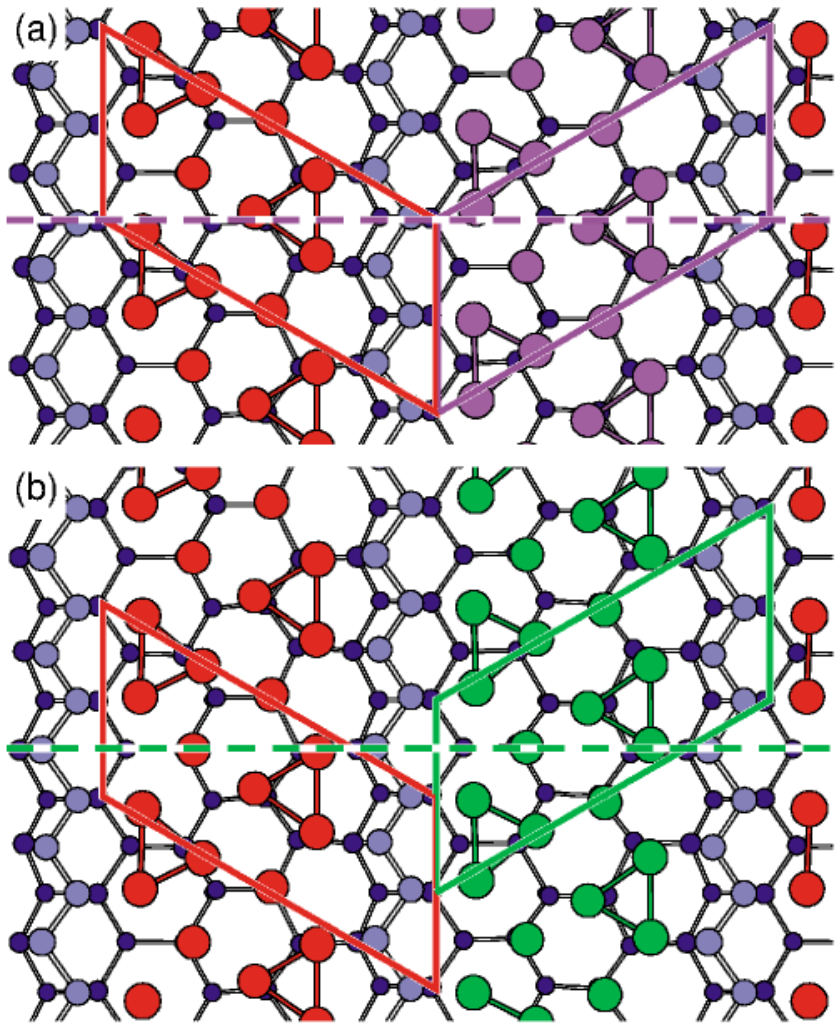

FIG. 4 (color). Illustration of the $(4 \times 2)$-subcell disorder. (a) shows the same structure as Fig. 2(a); green atoms in (b) are shifted by $[0,1]_{\text {LEED }}$. Details are explained in the text. 
in the chain direction. At lower temperature close to the transition, 2D (or 3D) correlations with arbitrary wave vectors are expected to break up the diffuse lines, which then condense into (isotropic) spots indicating the formation of a fully developed superstructure. The diffuse lines observed at high temperature normally have correlation lengths similar to that of the underlying surface lattice (i.e., widths similar to the fractional-order spots) $[1,6]$. For the phase transition under study a different behavior is observed. Around $20 \mathrm{~K}$ a good transverse correlation has developed giving rise to a doubling of the unit cell in the direction perpendicular to the chains. But even at $20 \mathrm{~K}$ along the chains streaks are observed with a scattering vector of $k=\frac{1}{2}$ indicating that the $(8 \times 2)$ superstructure is not fully developed and that the charge density fluctuations have not condensed. An analysis of the band structure indicates that a gap or pseudogap with that wave vector does exist [11]. The diffraction results are not compatible with a $1 \mathrm{D}$ CDW condensation, but the simple Peierls picture could be modified by the fact that three Fermi vectors are needed to describe this system [11].

Recently for another example of 1D self-assembled metallic wires on silicon, namely the $\operatorname{Si}(111)-(5 \times 1)$-Au reconstruction, a Luttinger-liquid-like behavior at low temperature was suggested [15], indicated by the lack of dispersion of the Au-induced state in the direction perpendicular to the chains and vanishing spectral intensity at the Fermi level. The same 1D metallic behavior and a Fermi-liquid to Luttinger-liquid transition has been suggested for the present system [12]. A simpler explanation for the driving force behind the phase transition would be the reduction in the total free energy due to the observed In-trimer formation and subsequent relaxation of the topmost $\mathrm{Si}$ atoms. Since the interactions within the adsorbate and between substrate and adsorbate cannot be described either in the idealized Peierls-picture or as a mere Fermi-to-Luttinger-liquid phase transition, further theoretical investigations are necessary to understand this complex system.

In summary, the low-temperature $\operatorname{Si}(111)-(8 \times 2)$-In reconstruction has been investigated by surface $\mathrm{x}$-ray diffraction and its structure has been solved. The phase transition is observed on cooling the $\mathrm{Si}(111)-(4 \times 1)$-In reconstruction to temperatures below $100 \mathrm{~K}$ and is accompanied by significant structural changes. A glide line occurs causing a doubling of the unit cell in the direction perpendicular to the indium chains and the periodicity along the chains doubles due to the outer indium atoms forming In trimers. Streaky half-order reflections are due to weak interchain correlation along the chains and indicate that the charge density fluctuations do not condense into a superstructure at $20 \mathrm{~K}$. The fact that at $20 \mathrm{~K}$ a strong transverse coupling is evidently established although charge density fluctuations at $k=\frac{1}{2}$ still persist is a clear indication that this system does not exhibit a simple quasi-1D CDW-driven transition. We hope that the structural model presented here will stimulate theoretical calculations and provide the basis for further investigations of the electronic properties of this fascinating system.

We thank A. B. McLean for useful discussions and the HASYLAB staff for technical assistance. Financial support from the Danish Research Council through Dansync, the German Bundesministerium für Bildung, Forschung und Technologie (BMBF Project No. 05 SE8 GUA6), the Deutsche Forschungsgemeinschaft Graduiertenkolleg "Physik nanostrukturierter Festkörper," the Volkswagen Stiftung, the European Community (TMR-Contract No. ERBFMGECT950059), and the IHP programme "Access to Research Infrastructures" of the European Commission (HPRI-CT-1999-00040) is gratefully acknowledged.

[1] G. Grüner, Density Waves in Solids (Addison-Wesley, Reading, MA, 1994).

[2] R. E. Peierls, Quantum Theory of Solids (Clarendon, Oxford, 1964).

[3] J. M. Luttinger, J. Math. Phys. (N.Y.) 4, 1154 (1963).

[4] A. Terrasi, M. Marsi, H. Berger, G. Margaritondo, R. J. Kelley, and M. Onellion, Phys. Rev. B 52, 5592 (1995).

[5] B. Dardel, D. Malterre, M. Grioni, P. Weibel, Y. Baer, and F. Lévy, Phys. Rev. Lett. 67, 3144 (1991).

[6] J. P. Pouget, J. Phys. IV (France) 10, Pr3-43 (2000).

[7] J. Kraft, M. G. Ramsey, and F. P. Netzer, Phys. Rev. B 55, 5384 (1997).

[8] J. J. Lander and J. Morrison, J. Appl. Phys. 36, 1706 (1965).

[9] O. Bunk, G. Falkenberg, J.H. Zeysing, L. Lottermoser, R. L. Johnson, M. Nielsen, F. Berg-Rasmussen, J. Baker, and R. Feidenhans'l, Phys. Rev. B 59, 12228 (1999).

[10] I. G. Hill, A. B. McLean, Phys. Rev. B 56, 15725 (1997); 59, 9791 (1999); Phys. Rev. Lett. 82, 2155 (1999).

[11] H. W. Yeom, S. Takeda, E. Rotenberg, I. Matsuda, K. Horikoshi, J. Schaefer, C. M. Lee, S. D. Kevan, T. Ohta, T. Nagao, and S. Hasegawa, Phys. Rev. Lett. 82, 4898 (1999).

[12] G. Le Lay, S. Hasegawa, V. Y. Aristov, L. Giovanelli, S. Takeda, H. Enriquez, V. Derycke, P. Soukiassian, R. Belkhou, and A. Taleb-Ibrahimi (to be published).

[13] See AIP Document No. EPAPS: E-PRLTAO-85-028049 for table containing parameter and data. This document may be retrieved via the EPAPS homepage (http://www.aip.org/pubservs/epaps.html) or from ftp.aip.org in the directory /epaps/. See the EPAPS homepage for more information.

[14] In order to reduce the degrees of freedom the silicon positions were optimized considering a Keating energy contribution. J. S. Pedersen, Surf. Sci. 210, 238 (1989).

[15] P. Segovia, D. Purdie, M. Hengsberger, and Y. Baer, Nature (London) 402, 504 (1999). 